\title{
Annual change of Pareto index dynamically deduced from the law of detailed quasi-balance
}

\author{
*Atushi Ishikawa \\ Kanazawa Gakuin University, Kanazawa 920-1392, Japan
}

\begin{abstract}
Employing data on the assessed value of land in 1983 - 2005 Japan, we investigate the dynamical behavior in the high scale region of non-equilibrium systems. From the detailed quasi-balance and Gibrat's law, we derive a relation between the change of Pareto index and a symmetry in the detailed quasi-balance. The relation is confirmed in the empirical data nicely.
\end{abstract}

PACS code : 04.60.Nc

Keywords : Econophysics; Pareto law; Gibrat law; Detailed quasi-balance

\section{Introduction}

In physics, universal laws in nature are extracted by analyzing empirical data, and various phenomena are explained by the laws. In econophysics, economic phenomena are investigated by using this physical approach [1]. From this point of view, recently a major progress has been made with the research in distributions of personal income or company size.

More than a hundred years ago, it is well known that a cumulative number $N(>x)$ obeys a power-law for $x$ which is larger than a certain threshold $x_{0}$ :

$$
N(>x) \propto x^{-\mu} \quad \text { for } \quad x>x_{0}
$$

This power-law and the exponent $\mu$ are called Pareto's law and Pareto index, respectively [2] ${ }^{1}$. Here $x$ is wealth, income, profits, assets, sales, the number of employees and etc.

Fujiwara et al. [5] have explained this phenomenon by using the law of detailed balance and Gibrat's law [6], which are observed in empirical data. The detailed balance is time-reversal symmetry:

$$
P_{12}\left(x_{1}, x_{2}\right)=P_{12}\left(x_{2}, x_{1}\right) .
$$

*e-mail address: ishikawa@kanazawa-gu.ac.jp

${ }^{1}$ Pareto's law is checked with high accuracy (See $[3,4]$ for instance). 
Here $x_{1}$ and $x_{2}$ are two successive incomes, profits, assets, sales, etc, and $P_{12}\left(x_{1}, x_{2}\right)$ is a joint probability distribution function (pdf). Gibrat's law states that the conditional probability distribution of growth rate $Q\left(R \mid x_{1}\right)$ is independent of the initial value $x_{1}$ :

$$
Q\left(R \mid x_{1}\right)=Q(R) .
$$

Here growth rate $R$ is defined as the ratio $R=x_{2} / x_{1}$ and $Q\left(R \mid x_{1}\right)$ is defined by using the pdf $P_{1}\left(x_{1}\right)$ and the joint pdf $P_{1 R}\left(x_{1}, R\right)$ as

$$
Q\left(R \mid x_{1}\right)=\frac{P_{1 R}\left(x_{1}, R\right)}{P_{1}\left(x_{1}\right)} .
$$

In the proof, they assume no model and only use these two underlying laws in empirical data.

Below the threshold $x_{0}$, it is also well known that the power-law is not observed [6, 7]. ${ }^{2} \mathrm{In}$ Ref. [11], by employing profits data of Japanese companies in 2002 and 2003 [12], Gibrat's law (3) is extended in the middle scale region as follows:

$$
\begin{array}{ll}
Q\left(R \mid x_{1}\right)=d R^{-t_{+}\left(x_{0}\right)-\alpha_{+} \ln \frac{x_{1}}{x_{0}}-1} & \text { for } R>1, \\
Q\left(R \mid x_{1}\right)=d R^{+t_{-}\left(x_{0}\right)-\alpha_{-} \ln \frac{x_{1}}{x_{0}}-1} & \text { for } R<1,
\end{array}
$$

where

$$
\begin{array}{rlrl}
\alpha_{+} \sim \alpha_{-} & \sim 0 & \text { for } x_{1}>x_{0}, \\
\alpha_{+} \sim \alpha_{-} & \neq 0 & \text { for } x_{\min }<x_{1}<x_{0}, \\
t_{+}\left(x_{0}\right)-t_{-}\left(x_{0}\right) & =\mu . & &
\end{array}
$$

By combining extended Gibrat's law (5) - (9) with the detailed balance (2), we have kinematically derived the following distribution function in the high and middle scale region uniformly

$$
P_{1}\left(x_{1}\right)=C x_{1}^{-(\mu+1)} e^{-\alpha \ln ^{2} \frac{x_{1}}{x_{0}}} \quad \text { for } x_{1}>x_{\min },
$$

where $\alpha=\left(\alpha_{+}+\alpha_{-}\right) / 2$.

All these findings are enormously important for the progress of econophysics. Above derivations are, however, valid only in the economic equilibrium where the detailed balance (2) holds. As the next step, the dynamics should be established. For this aim, we must investigate longterm economic data in which dynamical transitions are observed. Unfortunately, it is difficult to obtain personal income or company size data for a long period.

In Ref. [13], Kaizoji has reported that the distribution of Japanese land prices has similar features with one of personal income and company size. The database of the assessed value of land is available on the Ministry of Land, Infrastructure and Transport Government of Japanese World-Wide Web site [14].

In this paper, we analyze the high scale region data on the assessed value of land in a 23-year period $(1983$ - 2005) in order to investigate the dynamical behavior. As a result, we obtain a dynamical equation which non-equilibrium economic systems should satisfy.

\footnotetext{
${ }^{2}$ It is pointed out that the reason is the breakdown of Gibrat's law [5]. The breakdown of Gibrat's law in empirical data is reported by Stanley's group [8]. Takayasu et al. [9] and Aoyama et al. [10] also report that Gibrat's law does not hold in the middle scale region by using data of Japanese companies.
} 


\section{Data of land prices and the law of detailed quasi-balance}

In Japan, land is a very important asset which is distinguished from building. The transition of land prices has a possibility to influence the economy. In fact, Japanese economy experienced bubble term (1986 - 1991) caused by the abnormal rise of land prices.

The assessed value of land indicates the standard land prices evaluated by Ministry of Land, Infrastructure, and Transport. The investigation is undertaken on each piece of land assessed once a year. We employ the database of the assessed value of land covering the 23-year period from 1983 to 2005. The distributions of land prices are shown in Fig. 1, 2, 3, 4 and 5. The number of data points of land prices increases gradually, because the database only contains data points which exist in the 2005 evaluation.

From Fig. 1 - 5, the power-law is confirmed in the high scale region. For each year, we estimate Pareto index $\mu$ in the range of land prices from $2 \times 10^{5}$ to $10^{7}$ yen $/ \mathrm{m}^{2}$ where Pareto law holds approximately. Annual change of Pareto index $\mu$ from 1983 to 2005 is represented in Fig. 6. In this period, Pareto index has changed annually. This means that the system is not in equilibrium and the detailed balance (2) does not hold. Actually, the breakdown is observed in the scatter plot of all pieces of land assessed in the database (Fig. 7 and 8 for instance). There is no $x_{1} \leftrightarrow x_{2}$ symmetry in Fig. 7 and 8 obviously. On the other hand, the detailed balance ( $x_{1} \leftrightarrow x_{2}$ symmetry) is observed approximately in Fig. 9 and 10 for instance.

Here we make a simple assumption that the symmetry of the joint pdf $P_{12}\left(x_{1}, x_{2}\right)$ is represented as a regression line fitted by least-square method as follows

$$
\log _{10} x_{2}=\theta \log _{10} x_{1}+\log _{10} a
$$

The detailed balance (2) has the special symmetry, $\theta=a=1$. For each scatter plot, we measure $\theta, a$ and the result is shown in Fig. 11.

From this symmetry $\left(a x_{1}{ }^{\theta} \leftrightarrow x_{2}\right)$, we extend the detailed balance (2) to

$$
P_{12}\left(x_{1}, x_{2}\right)=P_{12}\left(\left(\frac{x_{2}}{a}\right)^{1 / \theta}, a x_{1}^{\theta}\right) .
$$

In this paper, we call this law the detailed quasi-balance.

\section{Dynamically varying Pareto index}

In this section, we derive the relation between the change of Pareto index $\mu$ and $\theta$ in Eq. (11) by extending the detailed balance (2) to the detailed quasi-balance (12). We assume Gibrat's law in the high scale region, because the number of data points is insufficient to observe Gibrat's law.

Due to the relation of $P_{12}\left(x_{1}, x_{2}\right) d x_{1} d x_{2}=P_{1 R}\left(x_{1}, R\right) d x_{1} d R$ under the change of variables from $\left(x_{1}, x_{2}\right)$ to $\left(x_{1}, R\right)$, these two joint pdfs are related to each other,

$$
P_{1 R}\left(x_{1}, R\right)=x_{1}{ }^{\theta} P_{12}\left(x_{1}, x_{2}\right),
$$


where we use a modified ratio $R \equiv x_{2} / x_{1}{ }^{\theta}$. From this relation, the detailed quasi-balance (12) is rewritten in terms of $P_{1 R}\left(x_{1}, R\right)$ as follows:

$$
P_{1 R}\left(x_{1}, R\right)=a R^{-1} P_{1 R}\left(\left(\frac{x_{2}}{a}\right)^{1 / \theta}, a^{2} R^{-1}\right) .
$$

Substituting the joint pdf $P_{1 R}\left(x_{1}, R\right)$ for the conditional probability $Q\left(R \mid x_{1}\right)$ defined in Eq. (4), the detailed quasi-balance is expressed as

$$
\begin{aligned}
\frac{P_{1}\left(x_{1}\right)}{P_{1}\left(\left(x_{2} / a\right)^{1 / \theta}\right)} & =\frac{a}{R} \frac{Q\left(a^{2} R^{-1} \mid\left(x_{2} / a\right)^{1 / \theta}\right)}{Q\left(R \mid x_{1}\right)} \\
& =\frac{a}{R} \frac{Q\left(a^{2} R^{-1}\right)}{Q(R)} \equiv G(a) .
\end{aligned}
$$

We have used Gibrat's law in Eq. (15).

By expanding Eq. (16) around $R=a$, the following differential equation is obtained

$$
a G^{\prime}(a) \theta P_{1}\left(x_{1}\right)+x_{1} P^{\prime}\left(x_{1}\right)=0 .
$$

The solution is given by

$$
P_{1}\left(x_{1}\right)=C_{1} x_{1}^{-a G^{\prime}(a) \theta}
$$

Here we consider two power-law distributions $N_{1}\left(>x_{1}\right) \propto x_{1}{ }^{\mu_{1}}$ and $N_{2}\left(>x_{2}\right) \propto x_{2}{ }^{\mu_{2}}$, which lead

$$
\begin{aligned}
& P_{1}\left(x_{1}\right)=C_{1} x_{1}^{-\mu_{1}-1}, \\
& P_{2}\left(x_{2}\right)=C_{2} x_{2}^{-\mu_{2}-1} .
\end{aligned}
$$

From Eq. (18) and (20), we identify that $a G^{\prime}(a) \theta=\mu_{1}+1$. On the other hand, by taking $x_{1} \longrightarrow\left(x_{2} / a\right)^{1 / \theta}$ transformation, Eq. (18) is rewritten as

$$
P_{1}\left(\left(\frac{x_{2}}{a}\right)^{1 / \theta}\right)=\frac{C_{1}}{a^{1 / \theta}} x_{2}^{-a G^{\prime}(a)}
$$

Identifying that $C_{2}=C_{1} / a^{1 / \theta}$ and $P_{1}\left(\left(x_{2} / a\right)^{1 / \theta}\right)=P_{2}\left(x_{2}\right)$, we obtain $a G^{\prime}(a)=\mu_{2}+1$. Consequently the relation between $\mu_{1}, \mu_{2}$ and $\theta$ is expressed as

$$
\frac{\mu_{1}+1}{\mu_{2}+1}=\theta
$$

This is the dynamical equation which non-equilibrium economic systems satisfy. We confirm that the empirical data satisfy this correlation in Fig. 12.

\section{Conclusion}

In this paper, we have investigated the dynamical behavior of non-equilibrium system in the high scale region by employing data on the assessed value of land in 1983 - 2005 Japan. By assuming the detailed quasi-balance (12) and Gibrat's law (3), we have derived a relation 
between the change of Pareto index and a symmetry in the detailed quasi-balance. The relation (22) has been confirmed in the empirical data nicely. The result in this paper is considered to be applied to other long-term economic data. The equation (22) is the dynamical one which non-equilibrium systems satisfy.

We should comment on two separations between $\theta$ and $\left(\mu_{1}+1\right) /\left(\mu_{2}+1\right)$ in Fig. 12. An abrupt jump of Pareto index between 1985 and 1986 (2001 and 2002) is observed in Fig. 6. This means that the system changes vigorously in this period, where the symmetry is not represented as Eq. (11). Nevertheless, the dynamical equation (22) is valid in almost all the other quasistatic periods.

The finding in this paper is a first step to explain varying macro phenomena by using laws in non-equilibrium systems. For the next step, we should investigate the dynamical behavior in the middle scale region.

\section{References}

[1] R.N. Mategna and H.E. Stanley, An Introduction to Econophysics, Cambridge University Press, UK, 2000.

[2] V. Pareto, Cours d'Economique Politique, Macmillan, London, 1897.

[3] H. Aoyama, W. Souma, Y. Nagahara, H.P. Okazaki, H. Takayasu and M. Takayasu, Fractals 8 (2000) 293;

W. Souma, Fractals 9 (2001) 463.

[4] A. Drăgulescu and V.M. Yakovenko, Physica A299 (2001) 213.

[5] Y. Fujiwara, W. Souma, H. Aoyama, T. Kaizoji and M. Aoki, Physica A321 (2003) 598;

H. Aoyama, W. Souma and Y. Fujiwara, Physica A324 (2003) 352;

Y. Fujiwara, C.D. Guilmi, H. Aoyama, M. Gallegati and W. Souma, Physica A335 (2004) 197 ;

Y. Fujiwara, H. Aoyama, C.D. Guilmi, W. Souma and M. Gallegati, Physica A344 (2004) 112 ;

H. Aoyama, Y. Fujiwara and W. Souma, Physica A344 (2004) 117.

[6] R. Gibrat, Les inegalites economiques, Paris, Sirey, 1932.

[7] W.W. Badger, in: B.J. West (Ed.), Mathematical Models as a Tool for the Social Science, Gordon and Breach, New York, 1980, p. 87;

E.W. Montrll and M.F. Shlesinger, J. Stat. Phys. 32 (1983) 209.

[8] M.H.R. Stanley, L.A.N. Amaral, S.V. Buldyrev, S. Havlin, H. Leschhorn, P. Maass, M.A. Salinger and H.E. Stanley, Nature 379 (1996) 804;

L.A.N. Amaral, S.V. Buldyrev, S. Havlin, H. Leschhorn, P. Maass, M.A. Salinger, H.E. Stanley and M.H.R. Stanley, J. Phys. (France) I7 (1997) 621;

S.V. Buldyrev, L.A.N. Amaral, S. Havlin, H. Leschhorn, P. Maass, M.A. Salinger, 
H.E. Stanley and M.H.R. Stanley, J. Phys. (France) I7 (1997) 635;

L.A.N. Amaral, S.V. Buldyrev, S. Havlin, M.A. Salinger and H.E. Stanley, Phys. Rev. Lett. 80 (1998) 1385;

Y. Lee, L.A.N. Amaral, D. Canning, M. Meyer and H.E. Stanley, Phys. Rev. Lett. 81 (1998) 3275 ;

D. Canning, L.A.N. Amaral, Y. Lee, M. Meyer and H.E. Stanley, Economics Lett. 60 (1998) 335 .

[9] H. Takayasu, M. Takayasu, M.P. Okazaki, K. Marumo and T. Shimizu, cond-mat/0008057, in: M.M. Novak (Ed.), Paradigms of Complexity, World Scientific, 2000, p. 243.

[10] H. Aoyama, 9th Annual Workshop on Economic Heterogeneous Interacting Agents (WEHIA 2004);

H. Aoyama, Y. Fujiwara and W. Souma, The Physical Society of Japan 2004 Autumn Meeting.

[11] A. Ishikawa, Derivation of the distribution from extended Gibrat's law, physics/0508178.

[12] TOKYO SHOKO RESEARCH, LTD., http://www.tsr-net.co.jp/.

[13] T. Kaizoji, Physica A326 (2003) 256.

[14] The Ministry of Land, Infrastructure and Transport Government of Japan's World-Wide Web site, http://nlftp.mlit.go.jp/ksj/.

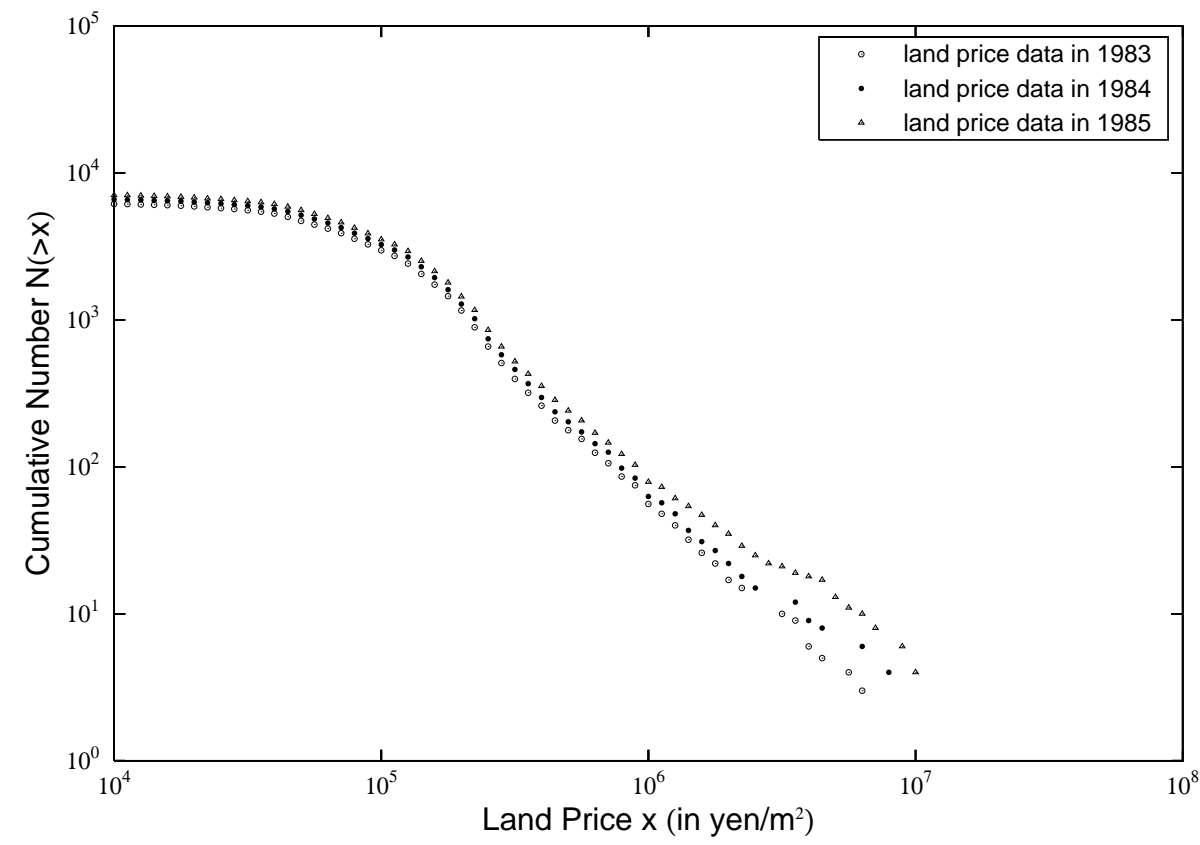

Figure 1: Cumulative number distribution $N(>x)$ of land prices in $1983-1985$. The number of the data points is "6,262", "6,706" and "7,169", respectively. 


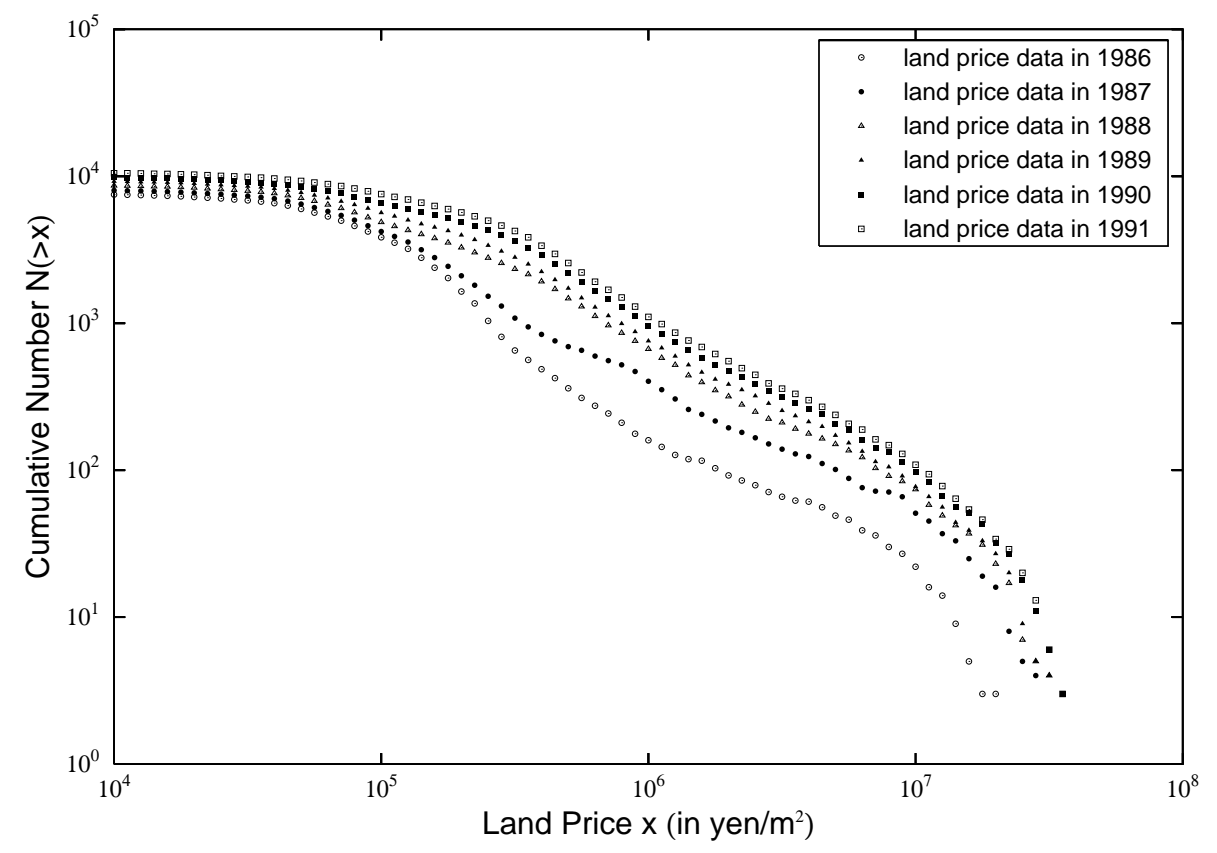

Figure 2: Cumulative number distribution $N(>x)$ of land prices in 1986 - 1991. The number of the data points is " 7,613 ", "8,120", 8,739", " $9,321 "$, " 9,927 " and " 10,603 ", respectively.

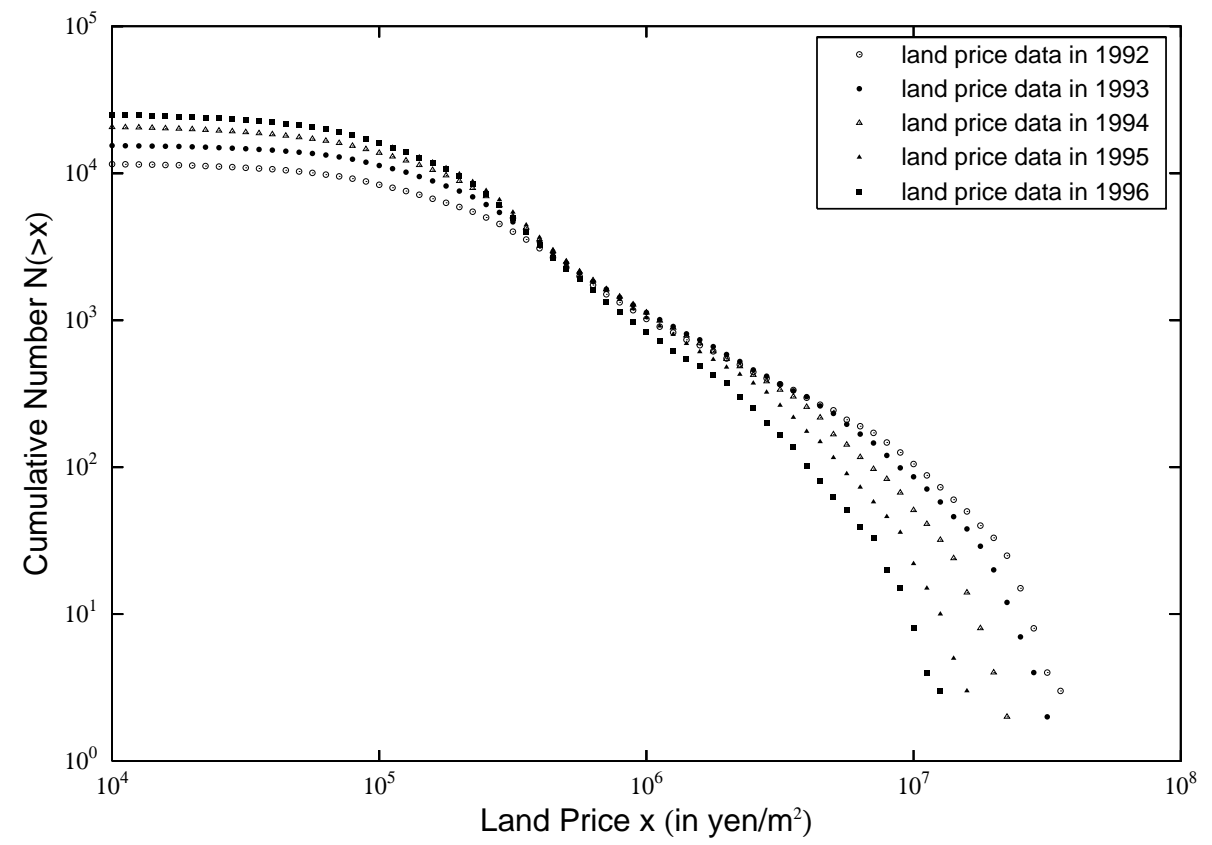

Figure 3: Cumulative number distribution $N(>x)$ of land prices in $1992-1996$. The number of the data points is " 11,641 ", " 15,538 ", “20,807", “24,753" and "25,091", respectively. 


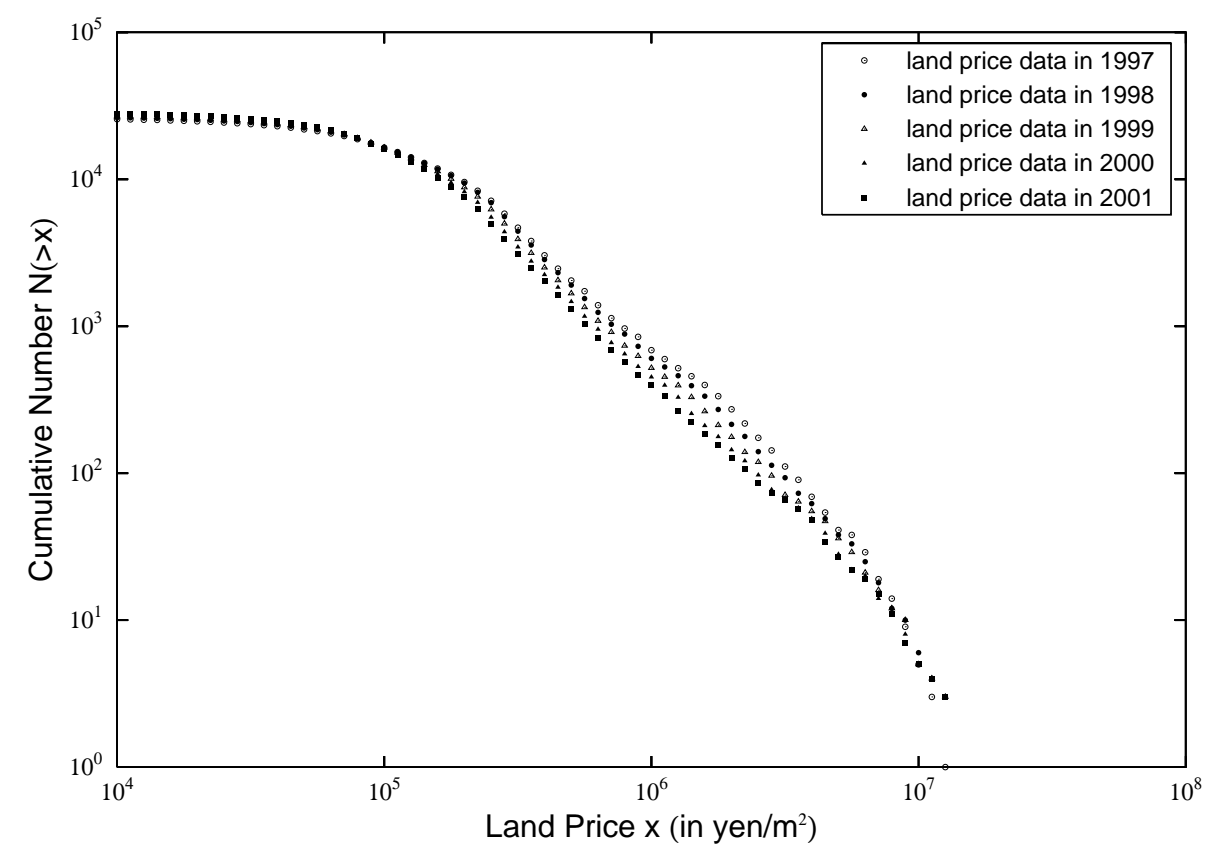

Figure 4: Cumulative number distribution $N(>x)$ of land prices in $1997-2001$. The number of the data points is " 25,845 ", " 26,524 ", " 27,073 ", " 27,738 " and " 28,294 ", respectively.

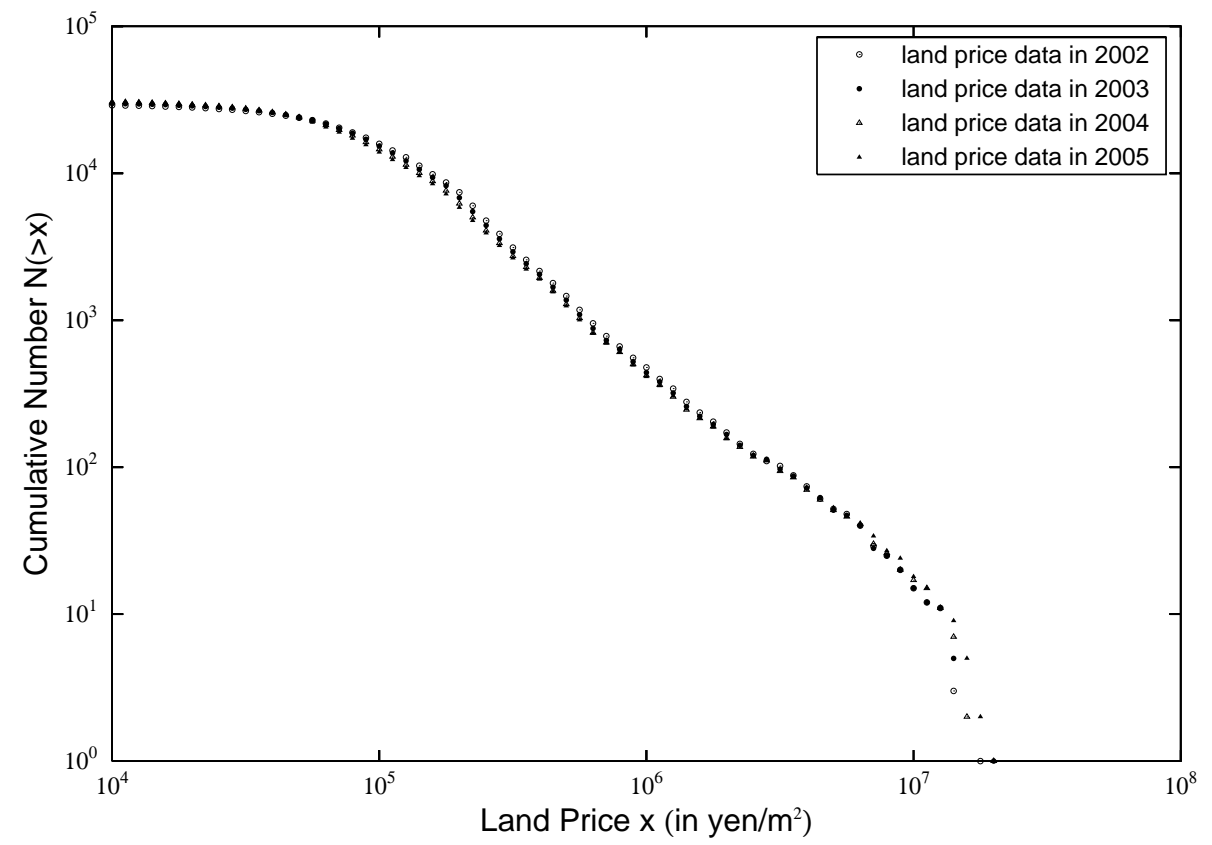

Figure 5: Cumulative number distribution $N(>x)$ of land prices in $2002-2005$. The number of the data points is " 29,279 ", " 30,138 ", " 30,775 " and " $31,230 "$, respectively. 


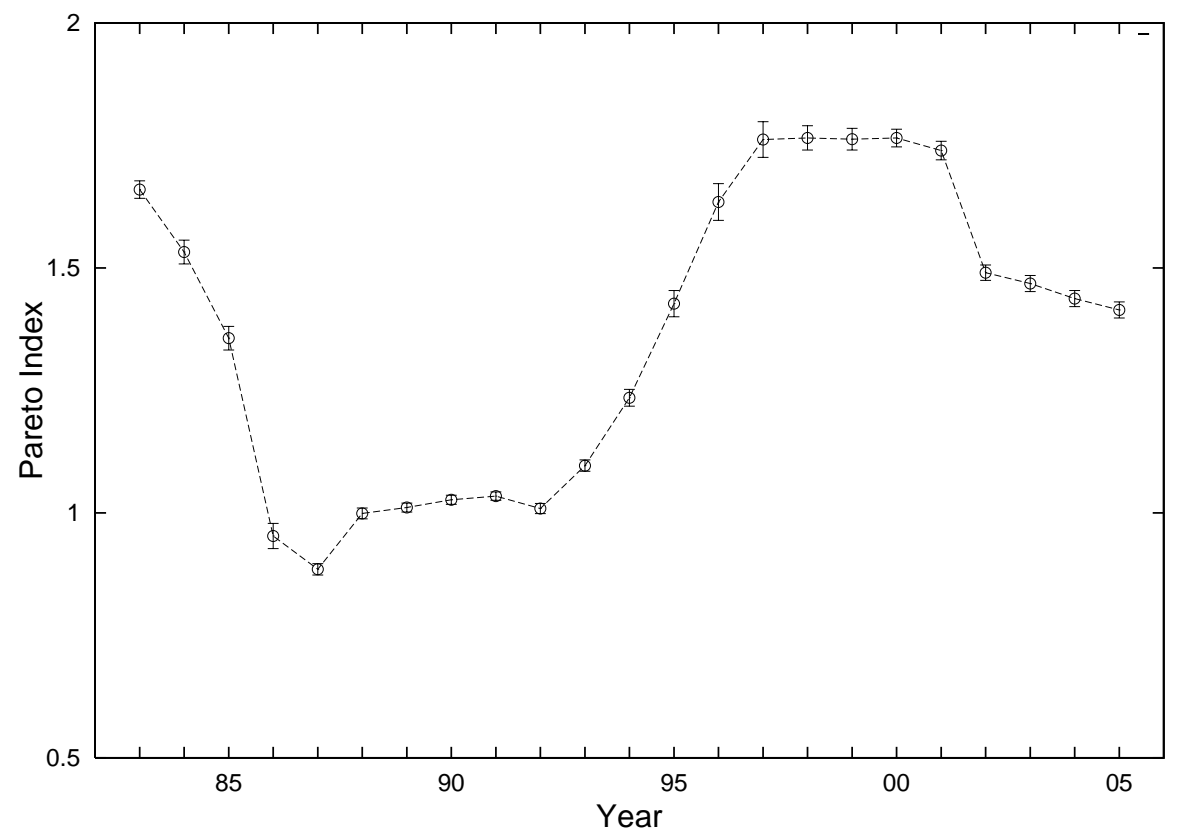

Figure 6: Annual change of Pareto index $\mu$ from 1983 to 2005.

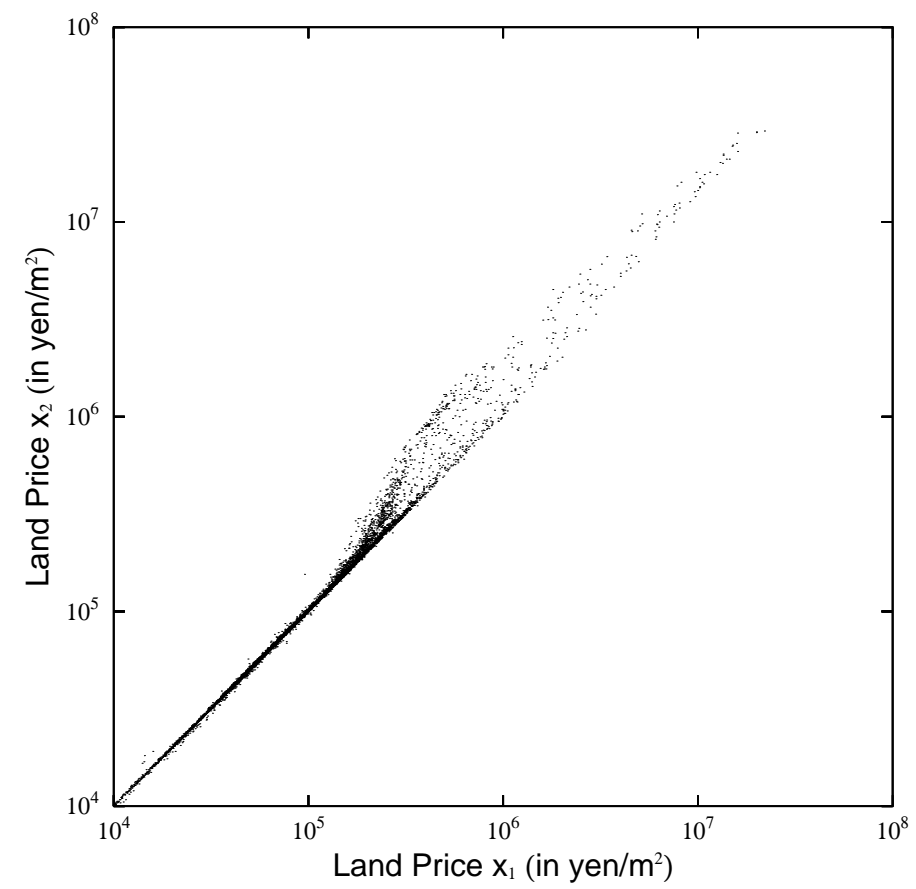

Figure 7: The scatter plot of all pieces of land assessed in the database, the values of which in $1986\left(x_{1}\right)$ and $1987\left(x_{2}\right)$ exceeded $10^{4}$ yen $/ \mathrm{m}^{2}$. The number of data points is " 1,603 ". 


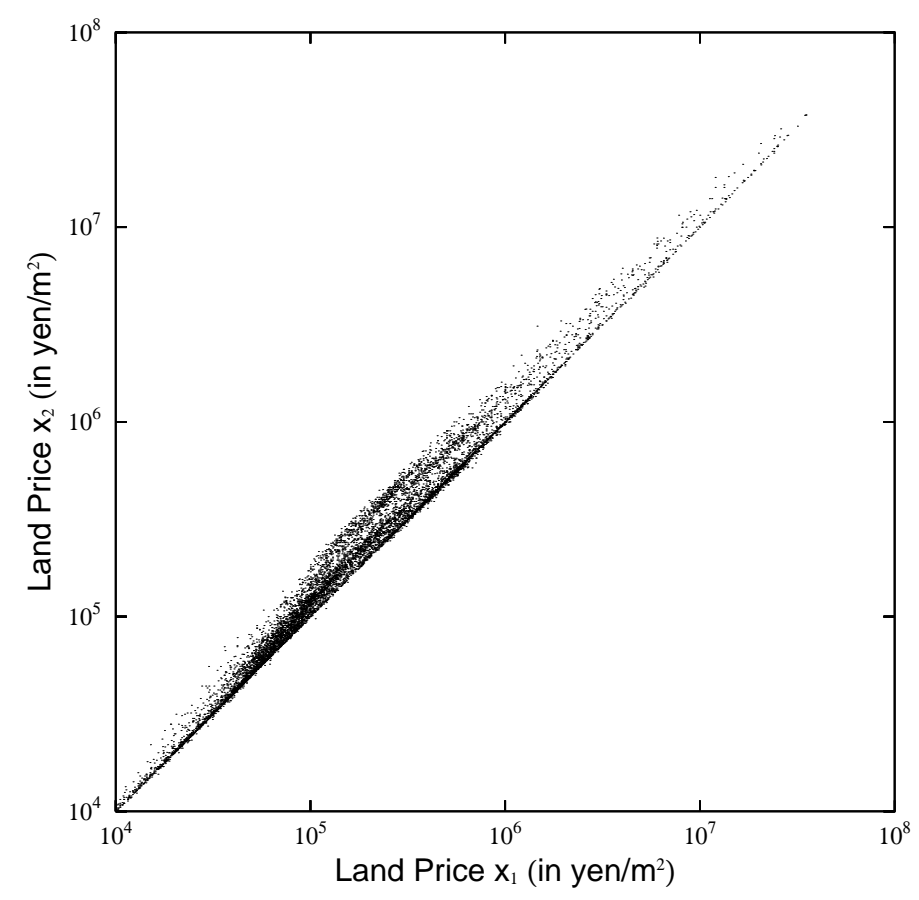

Figure 8: The scatter plot of all pieces of land assessed in the database, the values of which in $1989\left(x_{1}\right)$ and $1990\left(x_{2}\right)$ exceeded $10^{4}$ yen $/ \mathrm{m}^{2}$. The number of data points is " 3,886 ".

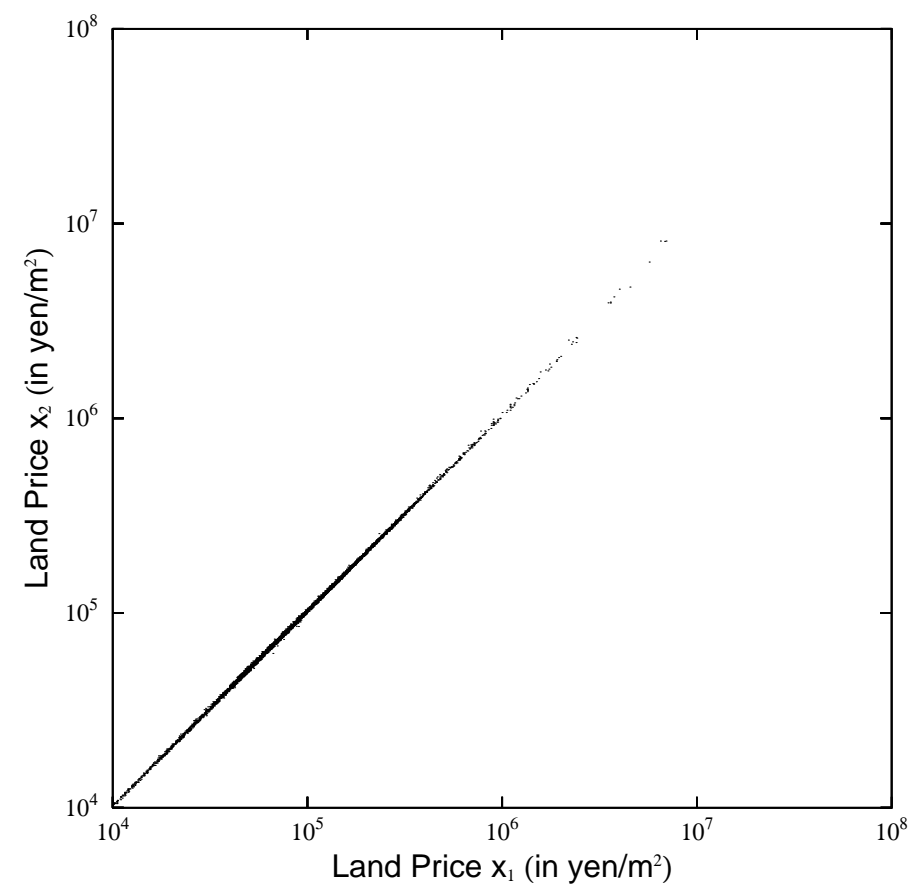

Figure 9: The scatter plot of all pieces of land assessed in the database, the values of which in $1983\left(x_{1}\right)$ and $1984\left(x_{2}\right)$ exceeded $10^{4}$ yen $/ \mathrm{m}^{2}$. The number of data points is "1,160". 


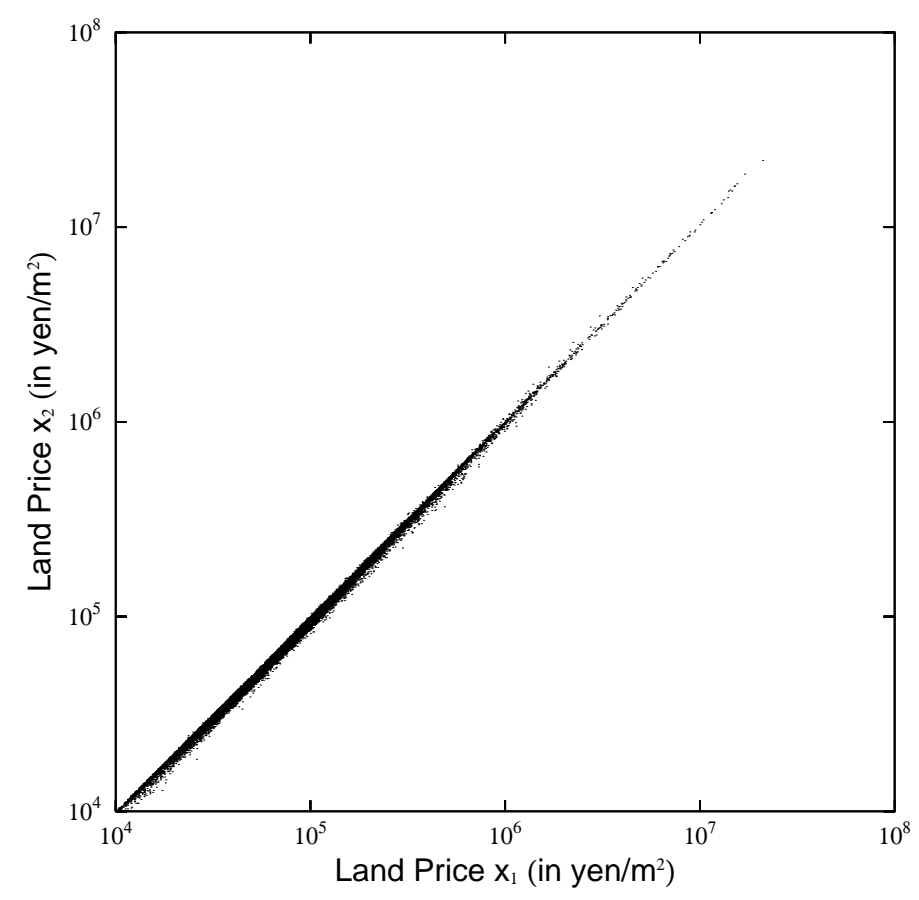

Figure 10: The scatter plot of all pieces of land assessed in the database, the values of which in $2004\left(x_{1}\right)$ and $2005\left(x_{2}\right)$ exceeded $10^{4}$ yen $/ \mathrm{m}^{2}$. The number of data points is " 5,739 ".

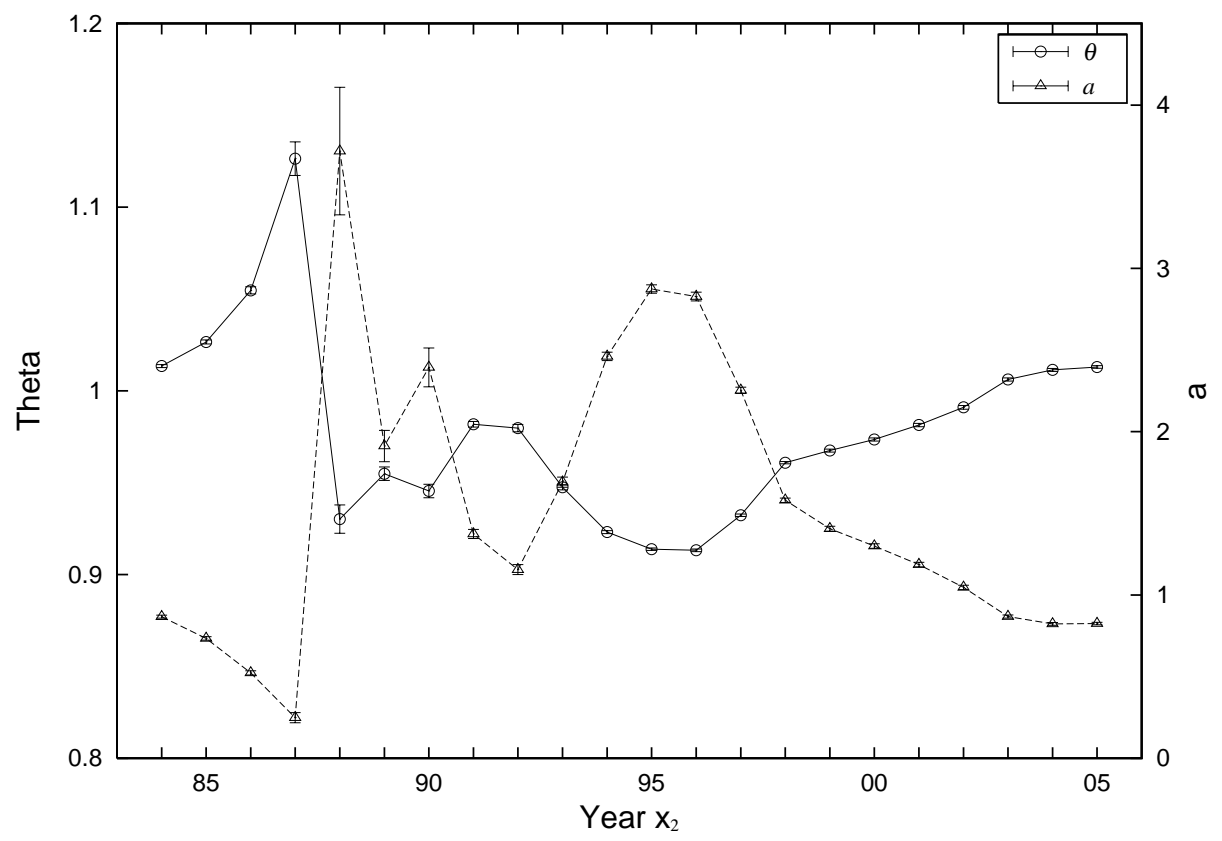

Figure 11: Annual change of $\theta$ and $a$ of Eq. (11) in the year $\left(x_{1}, x_{2}\right)=(1983,1984)-$ $-(2004,2005)$. 


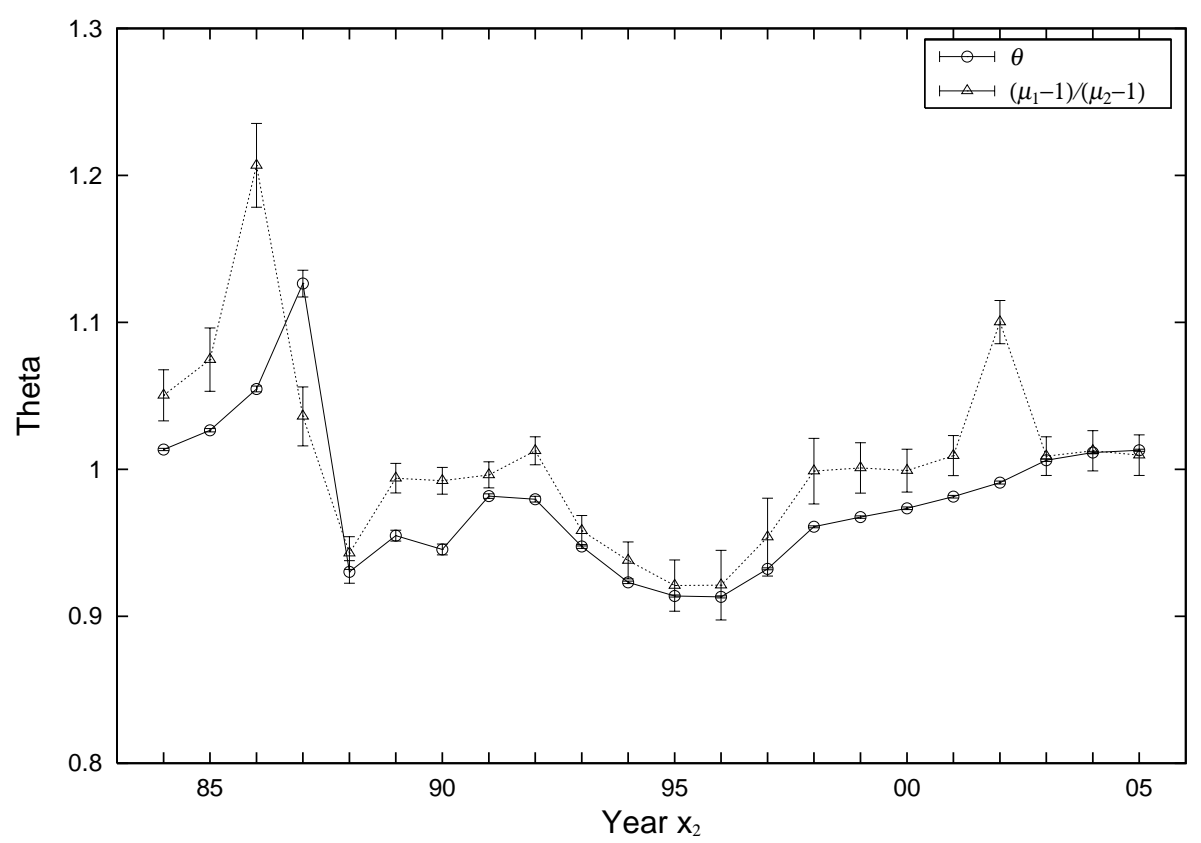

Figure 12: Annual change of $\theta$ and $\left(\mu_{1}+1\right) /\left(\mu_{2}+1\right)$ in the year $\left(x_{1}, x_{2}\right)=(1983,1984)-$ $-(2004,2005)$. 